\title{
Hábitos e comportamentos alimentares de adolescentes com sintomas de anorexia nervosa
}

\author{
Food habits and feeding behavior in adolescents with \\ symptoms of anorexia nervosa
}

Karin Louise Lenz DUNKER'

Sonia Tucunduva PHILIPPI ${ }^{2}$

\section{RESU M O}

Foram avaliados os hábitos e comportamentos alimentares de adolescentes do sexo feminino com sintomas de anorexia nervosa de uma escola particular. Foi identificada a presença de sintomas através do "Teste de Atitudes Alimentares". O recordatório de 24 horas "modificado" foi usado para avaliar os alimentos consumidos e um questionário foi aplicado para investigar as preferências e aversões alimentares. Das 279 alunas estudadas, $21,1 \%$ apresentaram sintomas. Os alimentos mais consumidos entre estas estudantes foram frutas, hortaliças, leite desnatado e bala, e os menos consumidos foram refrigerante, chocolate, massa e batata frita. Os alimentos de que "mais gostam" foram, em ordem decrescente: massas, carnes, hortaliças e doces; e os de que "menos gostam" foram: hortaliças, carnes e gorduras. Houve aversão maior por doces nas adolescentes com sintomas de anorexia nervosa. Concluiu-se ter havido um alto número de alunas com sintomas apresentando aversões alimentares semelhantes às de anoréxicas.

Termos de indexação: anorexia nervosa, adolescentes, hábito alimentar, comportamento alimentar.

\section{A B S T R A C T}

The food habits and feeding behavior of adolescent girls with symptoms of anorexia nervosa from a private school were assessed. The presence of symptoms was identified by the "Eating Attitudes Test". A 24-hour recall was used to evaluate the consumed food, and a questionnaire was applied to investigate the preferences and aversions. From the 279 girls studied, $21.1 \%$ presented symptoms. The foods most consumed by these adolescents were fruit, vegetables, skimmed milk and candies. The less eaten were soft drinks, chocolate,

\footnotetext{
1 Mestre em Nutrição Humana Aplicada, Curso Interunidades, Universidade de São Paulo.

2 Departamento de Nutrição, Faculdade de Saúde Pública, Universidade de São Paulo. Av. Dr. Arnaldo, 715, $2^{\circ}$ andar, 01246-904, São Paulo, SP, Brasil. Correspondência para/Correspondence to: S.T. PHILIPPI.
} 
pasta and fried potatoes. The kinds of food they liked most were, in decreasing order: pasta, meat, vegetables and sweets; and those which they liked least were: vegetables, meat and fat. There was a higher aversion to sweets in the adolescents with symptoms of anorexia nervosa. It was concluded that there was a great number of girls with symptoms presenting food aversions similar to those of anorexic people.

Index terms: anorexia nervosa, adolescents, food habits, feeding behavior.

\section{N T R O D U Ç Ã O}

A incidência de transtornos alimentares praticamente dobrou nestes últimos 20 anos. Especificamente em relação à anorexia, o número de casos novos por ano teve um aumento constante entre 1955 e 1984 em adolescentes de 10 a 19 anos. A prevalência de anorexia nervosa (AN) varia de $2 \%$ a $5 \%$ em mulheres adolescentes e adultas. Nos Estados Unidos é a terceira doença crônica mais comum entre adolescentes, só perdendo para a obesidade e a asma (Fischer et al., 1995).

O aumento da incidência coincide com a ênfase na magreza feminina como uma expressão de atração sexual. Atualmente a sociedade valoriza a atratividade e a magreza em particular, fazendo da obesidade uma condição altamente estigmatizada e rejeitada. A associação da beleza, sucesso e felicidade com um corpo magro tem levado as pessoas à prática de dietas abusivas e de outras formas não saudáveis de regular o peso (Killen et al., 1993).

A anorexia nervosa é caracterizada por uma restrição dietética auto-imposta, com um padrão alimentar "bizarro" e acentuada perda de peso, que está associada a um temor intenso de engordar e à má percepção corporal, e que deve ser diagnosticada por meio dos critérios do Manual Diagnóstico e Estatístico de Transtornos Mentais (DSM-IV) e da Classificação Internacional de Doenças (CID-10) (Fleitlich et al., 2000).

A doença normalmente tem início com um jejum progressivo, no qual primeiramente não são consumidos os alimentos calóricos, estendendo-se posteriormente a outros tipos de alimentos (Alvarenga, 1997).
O padrão e comportamento alimentar são muito irregulares na AN. De acordo com Philippi (1999), "entende-se como prática ou comportamento alimentar todas as formas de convívio com o alimento. Entendido como um processo, constitui um conjunto de ações realizadas com relação ao alimento, que têm início com o momento da decisão; disponibilidade; modo de preparar; utensílios usados; características, horários e divisão da alimentação nas refeições do dia, preferências e aversões, encerrando processo com o alimento sendo ingerido".

Conforme relataram Huse \& Lucas (1984), em seu estudo com 96 anoréxicas, 37\% tinham o hábito de jejuar e 40\% apresentavam peculiaridades nas preferências alimentares ou aversões específicas por alimentos ou grupos, como evitar carnes, doces e sobremesas, gorduras de adição (manteiga, molhos de salada e maionese), embora preferissem doces e sobremesas.

A restrição energética afeta o consumo de macronutrientes. A aversão aos carboidratos e gorduras é evidente, enquanto o consumo de proteínas normalmente está dentro da porcentagem que deve ser ingerida, apesar de o total ser inadequado e sua utilização comprometida pela ingestão energética insuficiente (Fischer et al., 1995).

A literatura cita como principais fatores etiológicos da anorexia: sexo, sendo nove vezes mais comum em mulheres do que em homens, e idade, sendo o início geralmente na adolescência (Castro \& Goldenstein, 1995; Fleitlich et al., 2000).

A partir destes dados, constata-se que a anorexia nervosa tem início praticamente na adolescência, mais especificamente entre 15 e 
18 anos. Nesta fase ocorrem mudanças marcantes na fisiologia e bioquímica, com um acúmulo pronunciado de gordura, especialmente nas mulheres. O ganho de gordura durante a adolescência cessa e até se reverte nos homens, enquanto nas mulheres continua a aumentar, chegando a um índice de gordura corporal de 27\% aos 16 anos (Castro \& Goldenstein, 1995).

Em vista destas mudanças, as adolescentes começam a ficar preocupadas com o aumento de peso e com a forma física. Entre os 12-16 anos, há um aumento na insatisfação com as medidas dos quadris e o emagrecimento torna-se um grande desejo (Castro \& Goldenstein, 1995).

$\mathrm{O}$ ato de restringir os alimentos tem início geralmente na adolescência, em resposta a uma má aceitação das mudanças corporais, principalmente do peso, e, associado a fatores psicológicos individuais e familiares e ao forte apelo sociocultural do culto à magreza, pode predispor a um transtorno alimentar.

As mudanças ocorridas com relação à percepção da alimentação e do peso, assim como comportamentos alimentares inadequados, podem ser identificadas antes mesmo de a doença se instalar, ou após seu surgimento, por meio de instrumento específico, o Teste de Atitudes Alimentares (EAT-26), que evidencia sintomas da anorexia nervosa em populações clínicas e não clínicas (Garner et al., 1982).

Na literatura internacional existem vários estudos de identificação de sintomas em populações, como os de Nasser (1994), Castro \& Goldenstein (1995) e Pastore et al. (1996). No Brasil só existem as pesquisas recentes de Nunes (1997) e Ribeiro (1999). No entanto, não existem trabalhos que descrevam os hábitos e comportamentos alimentares destes indivíduos.

O conhecimento do perfil da alimentação de adolescentes é importante para revelar comportamentos e hábitos sinalizadores de um dos principais sintomas da $\mathrm{AN}$, que é a restrição alimentar. Pode-se assim intervir com medidas preventivas em escolas, através de educação nutricional, promovendo mudanças nos conceitos e associações incorretas entre alimentos e peso.

Por todos estes fatores expostos, o presente trabalho propôs-se a identificar adolescentes do sexo feminino com sintomas de AN em uma instituição de ensino particular, descrevendo seus hábitos e comportamentos alimentares.

\section{CASUÍSTICA M MÉTODOS}

A pesquisa foi realizada em uma instituição de ensino particular localizada em região urbana e central, no município de São Paulo, SP, no período de 5/10/1998 a 5/11/1998.

Foram requisitadas a participarem da pesquisa adolescentes de ensino médio do sexo feminino que freqüentavam a aula de educação física. Aceitaram participar do estudo 288 alunas.

Foram considerados como critérios de exclusão do estudo: estudantes com menos de 15 anos ou mais de 18 anos completos, questionários não preenchidos corretamente, alunas que não entregaram no mínimo dois recordatórios alimentares de 24 horas e o questionário EAT-26.

Desse modo, a população de estudo ficou constituída por 279 adolescentes do sexo feminino com idades de 15 a 18 anos e 11 meses.

A identificação dos indivíduos com sintomas foi feita a partir de um questionário validado por Garner et al. (1982) em anoréxicas: o Teste de Atitudes Alimentares (EAT-26), também utilizado para identificar sintomas em populações, considerando-se nesta condição aqueles com escore maior ou igual a 20. Neste estudo foi utilizada a versão em português de Nunes et al. (1994). O questionário foi aplicado no segundo dia da pesquisa, antes ou após o preenchimento do inquérito alimentar.

Para avaliar os alimentos mais consumidos foi aplicado o recordatório alimentar de 24 horas "modificado" (Dunker \& Philippi, 1999). Ele foi preenchido pelas próprias alunas durante a aula 
de educação física, nas quadras ou em salas de aula, com a presença da pesquisadora, para eventuais dúvidas, em três dias alternados no decorrer da semana para refletir o consumo de dois dias da semana e um dia do fim de semana.

Para investigar as preferências e as aversões alimentares, assim como as respectivas justificativas das adolescentes, foi utilizado um questionário de comportamento alimentar com as seguintes questões: 1. Quais alimentos e preparações de que você mais gosta? Porquê? 2 . De quais alimentos e preparações você não gosta? Porquê? 3. Existe algum alimento(s) que você tenha aversão ou medo de comer? Porquê?

Os alimentos de cada questão foram agrupados, de acordo com os oito grupos alimentares da Pirâmide Alimentar (Philippi et al., 1999), em: $\left.1^{\circ}\right)$ cereais, pães, tubérculos e raízes; $2^{\circ}$ ) hortaliças; $3^{\circ}$ ) frutas; $4^{\circ}$ ) leite e produtos lácteos; $5^{\circ}$ ) leguminosas; $6^{\circ}$ ) carnes e ovos; $7^{\circ}$ ) óleos e gorduras; $8^{\circ}$ ) açúcares e doces.

Os inquéritos foram submetidos a crítica pelos autores para verificação da consistência das informações. Os dados de alimentos consumidos, preferências e aversões alimentares foram processados para a obtenção da freqüência absoluta e relativa nos dois grupos de estudo (alunas com e sem sintomas de AN). A freqüência relativa foi calculada tomando-se por base $\mathrm{O}$ número de registros diários.

Para a análise dos dados, foi utilizado o teste de qui-quadrado $\left(\chi^{2}\right)$, com o objetivo de

Tabela 1. Freqüencia absoluta e relativa (\%) dos alimentos mais consumidos segundo escore do EAT. São Paulo, SP, 1999.

\begin{tabular}{|c|c|c|c|c|}
\hline \multirow[b]{2}{*}{ Alimentos } & \multicolumn{2}{|c|}{$\mathrm{EAT}+$} & \multicolumn{2}{|c|}{ EAT- } \\
\hline & $n$ & $\%$ & $\mathrm{n}$ & $\%$ \\
\hline Arroz branco & 100 & 60,2 & 367 & 61,7 \\
\hline Alface & 71 & 42,8 & 159 & 26,7 \\
\hline Tomate & 68 & 41,0 & 131 & 22,2 \\
\hline Pão francês & 52 & 31,3 & 214 & 36,0 \\
\hline Leite tipo B & 66 & 39,8 & 307 & 51,6 \\
\hline Feijão & 50 & 30,1 & 145 & 24,4 \\
\hline Leite desnatado & 48 & 28,9 & - & - \\
\hline Bife & 39 & 23,5 & 110 & 18,5 \\
\hline Chocolate em pó & 39 & 23,5 & 194 & 32,6 \\
\hline Filé de frango & 33 & 19,9 & 84 & 14,1 \\
\hline Requeijão & 25 & 15,1 & 96 & 16,1 \\
\hline Suco de laranja & 25 & 15,1 & 106 & 17,8 \\
\hline Pão de forma & 24 & 14,5 & 107 & 18,0 \\
\hline Cenoura & 23 & 13,9 & - & - \\
\hline Maçã & 22 & 13,3 & - & - \\
\hline Banana & 21 & 12,7 & - & - \\
\hline Mamão papaia & 20 & 12,0 & - & - \\
\hline Bala drops de hortelã & 20 & 12,0 & - & - \\
\hline Queijo mussarela & 19 & 11,4 & 106 & 17,8 \\
\hline Manteiga & 19 & 11,4 & 97 & 16,3 \\
\hline Batata frita & - & - & 102 & 17,1 \\
\hline Macarrão ao sugo & - & - & 72 & 12,1 \\
\hline Açúcar & - & - & 144 & 24,2 \\
\hline Coca-cola & - & - & 125 & 21,0 \\
\hline Guaraná & - & - & 85 & 14,3 \\
\hline Chocolate em barra & - & - & 82 & 13,8 \\
\hline
\end{tabular}


avaliar as diferenças significativas de freqüência relativa dos grupos alimentares nas variáveis de comportamento alimentar (preferências e aversões) entre as alunas com e sem sintomas de AN.

Em todos os testes, fixou-se um $\alpha=5 \%$ $(p \leq 0,05)$ para o nível de rejeição da hipótese de nulidade, assinalando-se com um asterisco (*) valores significativos.

O processamento dos dados foi feito pelo software "Virtual Nutri" e para tabulação e análise de dados utilizou-se o Excel (versão 97) e Epi Info (versão 6,04b).

\section{RES U L T A D O S}

De acordo com o EAT-26, foram encontradas 59 alunas $(21,1 \%)$ com um escore positivo (EAT+). O restante da população $(78,9 \%, n=220)$ foi considerado como EAT- (sem sintomas), para avaliar diferenças entre os grupos.

$\mathrm{Na}$ avaliação dos alimentos e suas preparações, observam-se algumas diferenças entre os vinte mais consumidos nos dois grupos EAT (Tabela 1).

Conforme se constatou na análise do questionário de comportamento alimentar, os alimentos de que as alunas "mais gostam" foram semelhantes entre os grupos EAT e sem diferenças estatisticamente significativas (Tabela 2).

$\mathrm{Na}$ avaliação dos alimentos de que as adolescentes "não gostam" também não foram encontradas diferenças estatisticamente significativas (Tabela 3).

As aversões alimentares diferem um pouco entre os grupos (Tabela 4), observando-se diferença estatisticamente significativa quanto a aversão por açúcares e doces $\left(\chi^{2}=4,12 ; p=0,04\right)$.

Em ambos os grupos os alimentos parecem ser evitados em virtude das suas características, como sabor (Ex.: gosto ruim, sem gosto, gosto amargo) e aparência (Ex.: nojento, meloso, gosmento, estranho), ou ainda por causar sensações (Ex.: passei mal, causa ânsia de vômito); no entanto, no grupo EAT+ constatou-se uma maior associação dos alimentos com o medo de engordar.

\section{I S C U S S Ã O}

No presente trabalho foram encontradas 59 alunas, ou seja, 21,1\% da população, com um escore positivo no EAT. Ao comparar esta porcentagem com outros estudos de países ocidentais e orientais com adolescentes de escolas públicas ou privadas, aplicando a mesma metodologia de identificação, pode-se notar que ela foi muito mais alta. Nasser (1994) encontrou $11,4 \%$ com escore positivo no Egito, Wlodarczyk-Bisaga \& Dolan (1996) obtiveram 12,6\% na Polônia e Pastore et al. (1996) tiveram como resultado $15 \%$.

O EAT é um teste validado e confiável em indivíduos com AN, o qual permite menores erros estatísticos, possibilitando comparações transculturais em relação às condutas alimentares das populações estudadas. Entretanto, por causa da grande variabilidade de valores, para se obter resultados mais adequados, o instrumento deve sempre ser adaptado às atitudes e hábitos dos diversos grupos culturais (Ortega et al., 1995). Segundo Williams (1987), o valor preditivo do EAT, ou seja, a probabilidade de a doença desenvolver-se em uma pessoa com um escore positivo, é baixo (somente 0,19 ). Isto significa que em cada 100 escores altos, somente 19 poderão vir a ter realmente a doença.

Conforme se observou ao analisar os vinte alimentos mais consumidos, as alunas do grupo EAT+ tinham algumas características de consumo diferentes do restante da população, mas que são comuns na adolescência.

No estudo de Doyle \& Feldman (1997) com adolescentes de escolas particulares do Amazonas, os autores verificaram que os alimentos preferidos para se "beliscar" foram, em ordem decrescente: refrigerantes, sucos de frutas, massas, frutas, doces, bolo, sorvete, biscoitos e batata frita. 
Tabela 2. Freqüencia absoluta e relativa (\%) dos alimentos de que "mais gostam" segundo escore do EAT e grupos alimentares. São Paulo, SP, 1999

\begin{tabular}{|c|c|c|c|c|}
\hline \multirow[b]{2}{*}{ Grupos alimentares } & \multicolumn{2}{|c|}{ EAT+ } & \multicolumn{2}{|c|}{ EAT- } \\
\hline & $n$ & $\%$ & $\mathrm{n}$ & $\%$ \\
\hline Cereais, pães, tubérculos, raízes & 58 & 28,4 & 207 & 28,2 \\
\hline Hortaliças & 37 & 18,1 & 127 & 17,3 \\
\hline Frutas & 13 & 6,4 & 47 & 6,4 \\
\hline Leite e produtos lácteos & 4 & 2,0 & 11 & 1,5 \\
\hline Leguminosas, nozes/oleaginosas & 10 & 4,9 & 24 & 3,3 \\
\hline Carnes e ovos & 47 & 23,0 & 161 & 22,0 \\
\hline Óleos e gorduras & 2 & 0,5 & 12 & 1,6 \\
\hline Açúcares e doces & 29 & 14,2 & 106 & 14,5 \\
\hline Outros $^{(* *)}$ & 5 & 2,5 & 38 & 5,2 \\
\hline Total & 205 & 100,0 & 733 & 100,0 \\
\hline
\end{tabular}

${ }^{(*)}=\operatorname{significante}(p \leq 0,05) ;{ }^{(*)}=$ Sanduíches (natural/fast-food); Comidas típicas (alemã, chinesa, árabe, e outras).

Tabela 3. Freqüencia absoluta e relativa (\%) dos alimentos de que "não gostam" segundo escore do EAT e grupos alimentares. São Paulo, SP, 1999

\begin{tabular}{|c|c|c|c|c|}
\hline \multirow[b]{2}{*}{ Grupos alimentares } & \multicolumn{2}{|c|}{ EAT+ } & \multicolumn{2}{|c|}{ EAT- } \\
\hline & $n$ & $\%$ & $n$ & $\%$ \\
\hline Cereais, pães, tubérculos, raízes & 5 & 5,0 & 31 & 7,7 \\
\hline Hortaliças & 37 & 36,6 & 150 & 37,1 \\
\hline Frutas & 5 & 5,0 & 20 & 5,0 \\
\hline Leite e produtos lácteos & 1 & 1,0 & 8 & 2,0 \\
\hline Leguminosas, nozes/oleaginosas & 4 & 4,0 & 19 & 4,7 \\
\hline Carnes e ovos & 36 & 35,6 & 139 & 34,4 \\
\hline Óleos e gorduras & 7 & 6,9 & 20 & 5,0 \\
\hline Açúcares e doces & 5 & 5,0 & 11 & 2,7 \\
\hline Outros ${ }^{(* *)}$ & 1 & 1,0 & 6 & 1,5 \\
\hline Total & 101 & 100,0 & 404 & 100,0 \\
\hline
\end{tabular}

${ }^{(*)}=$ significante $(p \leq 0,05) ;{ }^{* *)}=$ Sanduíches (natural/fast-food); Comidas típicas (alemã, chinesa, árabe, e outras).

Tabela 4. Freqüencia absoluta e relativa (\%) das aversões alimentares segundo escore do EAT e grupos alimentares. São Paulo, SP, 1999.

\begin{tabular}{|c|c|c|c|c|}
\hline \multirow[b]{2}{*}{ Grupos alimentares } & \multicolumn{2}{|c|}{ EAT+ } & \multicolumn{2}{|c|}{ EAT- } \\
\hline & $\mathrm{n}$ & $\%$ & $\mathrm{n}$ & $\%$ \\
\hline Cereais, pães, tubérculos, raízes & 2 & 4,9 & 5 & 3,6 \\
\hline Hortaliças & 6 & 14,6 & 28 & 20,0 \\
\hline Frutas & - & - & 5 & 3,6 \\
\hline Leite e produtos lácteos & - & - & 2 & 1,4 \\
\hline Leguminosas, nozes/oleaginosas & 1 & 2,4 & 4 & 2,9 \\
\hline Carnes e ovos & 19 & 46,3 & 75 & 53,6 \\
\hline Óleos e gorduras & 3 & 7,3 & 7 & 5,0 \\
\hline Açúcares e doces & 8 & $19,5^{*}$ & 10 & 7,1 \\
\hline Outros ${ }^{(* *)}$ & 2 & 4,9 & 4 & 2,9 \\
\hline Total & 41 & 100,0 & 140 & 100,0 \\
\hline
\end{tabular}

${ }^{(*)}=$ significante $(p \leq 0,05) ;{ }^{(* *)}=$ Sanduíches (natural/fast-food); Comidas típicas (alemã, chinesa, árabe, e outras). 
No entanto, na lista dos mais consumidos entre as alunas com sintomas apareceram alguns alimentos distintos, os quais, por suas propriedades, espelham a preocupação em fazer uma dieta equilibrada. Além dos alimentos consumidos pelo EAT-, há também o consumo de leite desnatado, de uma maior variedade de frutas e de hortaliças, enquanto não aparecem na relação gêneros alimentícios considerados energéticos, como batata frita, macarrão, refrigerantes, chocolate em barra. A freqüência de consumo de balas por este grupo, ocorrência não encontrada no EAT-, poderia sugerir que estas adolescentes utilizam esta guloseima como forma de suprimir a fome ou, ainda, "enganar o estômago".

A análise dos alimentos mais consumidos indica uma maior preocupação com a alimentação por parte das alunas EAT+. Outro fator a ser considerado seria a forma de preenchimento dos inquéritos, pois elas demonstraram ser mais específicas nas quantidades e qualidade dos produtos consumidos. Estas características de comportamento são comuns na $\mathrm{AN}$, onde há um perfeccionismo evidente e uma preocupação de estar ingerindo alimentos menos calóricos para perder ou manter peso.

A preocupação com alimentação encontrada nesta população pode ser considerada uma característica comum das adolescentes. De acordo com um estudo recente realizado em Porto Alegre com estudantes secundaristas, as mulheres demonstram ser mais críticas em relação à qualidade e ao padrão de sua alimentação do que os homens (Feijó et al., 1997).

Na avaliação dos alimentos de que o grupo EAT+ "mais gosta", constatou-se serem estes semelhantes ao EAT-. Houve maior preferência por massas, carnes, hortaliças, açúcares e doces. Apesar de as alunas EAT+ afirmarem gostar destes alimentos, na análise dos mais consumidos não foi constatado um consumo de massas, açúcares e doces, como no EAT-. Esta poderia ser mais uma característica que diferencia este grupo e se assemelha ao comportamento anoréxico, como discutido por Huse \& Lucas (1984), os quais relataram uma evidente negação por doces e massas na AN.

Ao observar os alimentos de que a população "não gosta", encontram-se, em ordem decrescente, aqueles dos grupos de hortaliças, carnes e ovos, e gorduras. Este resultado deve-se ao fato de a maioria dos gêneros alimentícios citados serem considerados tabus para a sociedade, ou causadores de mal à saúde. Como exemplos, podem ser citados: entre as hortaliças, repolho, agrião, espinafre, jiló, quiabo, chuchu, berinjela, beterraba, abobrinha, pimentão, abóbora, rabanete, nabo; entre as carnes, pescados, vísceras (fígado); entre os moluscos/crustáceos, mariscos, lula, camarão; e entre as gorduras, as frituras.

Priore (1998), em seu estudo com adolescentes dos sexos masculino e feminino, encontrou maior preferência por refrigerantes, massas, sucos, doces, carnes, batatas, frutas, sanduíches, hortaliças. Foram citados como recusas alimentares: hortaliças, carnes e ovos, frutas/sucos/refrescos, leite e derivados, leguminosas, tubérculos e, por último, os doces.

A avaliação das aversões alimentares atingiu em pequeno grau seus objetivos, pois a maioria das alunas associou a aversão ao não gostar devido à aparência, à textura e ao sabor dos alimentos, e não ao medo de engordar, causa que o instrumento buscava identificar. Apesar deste erro de interpretação, foram observadas algumas diferenças entre os grupos. Ambos tinham aversões semelhantes, mas o grupo EAT+, apresentou uma não muito comum na adolescência e estatisticamente significativa: a freqüência relativa de aversão por açúcares e doces ultrapassou o dobro da obtida no EATCoincidentemente, neste grupo, encontrou-se maior número de alunas que tinham o medo de engordar como justificativa para a aversão.

Os dados de hábitos e comportamentos alimentares obtidos nesta pesquisa podem ser comparados ao estudo de Binsbergen et al. (1988), no qual estes analisaram as preferências e aversões alimentares de mulheres anoréxicas e de não anoréxicas. Segundo constataram os 
autores, aquelas freqüentemente têm uma maior aversão por alimentos doces (açúcar, chocolate, refrigerantes, e outros), em comparação com estas. Eles também observaram que as anoréxicas tendem a ter uma menor preferência por alimentos fontes de carboidratos e gorduras, considerados calóricos, tais como: batata, batata frita, pão branco, arroz, leite integral. A exclusão de alimentos como pães e cereais, gordura e açúcar também foi encontrada em adolescentes com comportamentos alimentares inadequados na pesquisa de Martin et al. (1999).

Conforme relata o estudo realizado em São Paulo, no ambulatório de adolescência clínica do Núcleo de Nutrição, Alimentação e Desenvolvimento Infantil (NUNADI) do Centro de Referência da Saúde da Mulher (CRSM), é característico desta fase gostar mais de massas e doces do que de hortaliças, como verificado no presente trabalho. Há também registros de algumas aversões semelhantes por pescados, bacalhau, fígado, carne suína, frutos do mar, hortaliças (beterraba, berinjela, pimentão, jiló, repolho, agrião) (Grazini et al., 1995).

A partir destes dados, pode-se afirmar que as adolescentes têm uma percepção de adequação alimentar, pois dizem gostar de alimentos fontes de carboidratos (massas), proteínas (carnes), e vitaminas e minerais (hortaliças), e buscam evitar as gorduras, o que seria o perfil de uma alimentação equilibrada. No entanto, no grupo com sintomas podem-se verificar aversões distintas e muito semelhantes às das anoréxicas.

De acordo com Fletcher et al. (1989), o estudo deve ser considerado de validade interna, pois se aplica a condições particulares de um grupo populacional e, portanto, seus resultados não podem ser extrapolados para a população brasileira em geral.

\section{ONCLUSÃ O}

Os hábitos alimentares das alunas com sintomas demostraram ser diferentes daquelas sem sintomas, com a presença de alimentos mais saudáveis. O comportamento alimentar mostrou ser característico da adolescência, mas a aversão aos doces foi significativa e semelhante àquela apresentada na anorexia.

O número elevado de jovens com sintomas de anorexia nervosa encontrado neste estudo e o aumento da prevalência de adolescentes com anorexia nervosa, segundo dados da literatura, são fatores importantes para justificar a implementação de programas de intervenção nutricional.

Poderiam ser recomendadas medidas como: implementar políticas na área de educação e saúde, visando prevenir o aparecimento da doença, por meio de campanhas governamentais veiculadas pela mídia; incluir no currículo o conteúdo programático em nutrição, alimentação e padrões de peso e a atividade física desde a pré-escola; envolver e sensibilizar as empresas alimentícias para veicularem mensagens de alimentação adequada nos rótulos dos alimentos; envolver a família dos adolescentes, motivando-os com reuniões e discussões nas escolas sobre a importância dos hábitos e comportamentos alimentares.

REF E R Ê NCIAS B I B LIOG RÁ F I C A S

ALVARENGA, M.S. Anorexia nervosa e bulimia nervosa: aspectos nutricionais. São Paulo, 1997. Dissertação (Mestrado em Nutrição Humana Aplicada) - Curso Interunidades, Universidade de São Paulo, 1997.

BINSBERGEN, S.J.M., HULSHOF, K.F.A.M., WEDEL, M., ODINK, J., BENNINK, H.J.T. Food preferences and aversions and dietary pattern in anorexia nervosa patients. European Journal of Clinical Nutrition, London, v.42, n.8, p.671-678, 1988.

CASTRO, J.M., GOLDENSTEIN, S. Eating Attitudes and behaviors of pre- and postpubertal females: clues to the etiology of eating disorders. Physiology \& Behavior, v.58, n.1, p.15-23, 1995.

DOYLE, E.I., FELDMAN, R.H.L. Factors affecting nutrition behavior among middle-class 
adolescents in urban area of Northern Region of Brazil. Revista de Saúde Pública, São Paulo, v.31, n.4, p.342-50, 1997.

DUNKER, K.L.L., PHILIPPI, S.T. Recordatório alimentar de 24 horas "modificado" avaliação do consumo alimentar de adolescentes. In: CONGRESSO LATINO-AMERICANO DE NUTRIÇÃO HUMANA, 1., 1999, Gramado. Brasil. Anais... Gramado : SLANH, 1999. p.65

FEIJÓ, R.B., SUKSTER, E.B., FRIEDRICH, L., FIALHO, L., DZIEKANIAK, K.S., CHRISTINI, P.W., MACHADO, L.R., GOMES, K.V., CARDOSO, I.H. Estudo de hábitos alimentares em uma amostra de estudantes secundaristas de Porto Alegre. Pediatria (São Paulo), v.19, n.4, p.257-262, 1997.

FISCHER, M., GOLDEN, N.H., KATZMAN, D.K., KREIPE, R.E., REES, J., SHEBENDACH, J., SIGMAN, G., AMMERMAN, S., HOBEREMAN, H.M. Eating disorders in adolescents: a background paper. Journal of Adolescent Health Care, New York, v.16, n.6, p.420-437, 1995.

FLEITLICH, B.W., LARINO, M.A., COBELO, A., CORDÁS, T.A. Anorexia nervosa na adolescência. Jornal de Pediatria, Rio de Janeiro, v.76, n.3, p.323-329, 2000.

FLETCHER, R.H, FLETCHER, S.W., WAGNER, E.H. Epidemiologia clínica: bases científicas da conduta médica. Porto Alegre : Artes Médicas, 1989. p.32.

GARNER, D.M., OLMSTED, M.P., BOHR, Y., GARFINKEL, P.E. The Eating Attitudes Test: psychometric features and clinical correlates. Physiological Medicine, v.12, p.871-878, 1982.

GRAZINI, J.T., VITALLE, M.S.S., MEDEIROS, S., BRASIL, A.L.D., MORAES, D.E.B., ASSIS, A.N., NÓBREGA, F.J. Modelo de inquérito para investigação de hábitos, preferência e aversões alimentares de adolescentes. Revista Paulista de Pediatria, v.13, n.1, p.10-14, 1995.

HUSE, D.M., LUCAS, A.R. Dietary patterns in anorexia nervosa. American Journal of Clinical Nutrition, Bethesda, v.40, n.2, p.251-254, 1984.

KILLEN, J.D., TAYLOR, C.B., HAMMER, L.D., LITT, I., WILSON, D.M., RICH, T., HAYARD, C., SIMMONDS,
B., KRAEMER, H., VARADY, A. An attempt to modify unhealthful eating attitudes and weight regulation practices of young adolescent girls. International Journal of Eat Disorders, v.13, n.4, p.369-384, 1993.

MARTIN, A.R., NIETO, J.M., JIMENEZ, M.A., RUIZ, J.P., VAZQUEZ, M.C., FERNANDEZ, Y.C., GOMEZ, M.A., FERNANDEZ, C.C. Unhealthy eating behaviour in adolescents. European Journal of Epidemiology. v.15, n.7, p.643-648, 1999

NASSER, M. Screening for abnormal eating attitudes in a population of Egyptian secondary school girls. Social Psychiatry and Psychiatric Epidemiology, Berlin, v.29, n.1, p.25-30, 1994.

NUNES, M.A., BAGATINI, L.F., ABUCHAIM, A.L., KUNZ, A., RAMOS, D., SILVA, A., SOMENZI, L., PINHEIRO, A. Distúrbios da conduta alimentar: considerações sobre o Teste de Atitudes Alimentares (EAT). Revista ABP-APAL, v. 16, n.1, p.7-10, 1994.

NUNES, M.A. Prevalência de comportamentos alimentares anormais e práticas inadequadas de controle de peso em mulheres de 12 a 29 anos em Porto Alegre. Pelotas, 1997. Dissertação (Mestrado) - Universidade Federal de Pelotas, 1997.

ORTEGA, R.M., REQUEJO, A.M., ANDRÉS, P., LÓPEZ-SOBALER, A.M., REDONDO, R., GONZÁLEZ- FERNÁNDEZ, A.M. Relationship between diet composition and body mass index in a group of Spanish adolescents. British Journal of Nutrition, London, v.74, n.6, p.765-773, 1995.

PASTORE, D.R., FISCHER, M., FRIEDMAN, S.B. Abnormalities in weight status, eating attitudes and eating behaviors among urban high school students: correlations with self-esteem and anxiety. Journal of Adolescent Health, New York, v. 18, n.5, p.312-319, 1996.

PHILIPPI, S.T. Guia alimentar para o ano 2000. In: ANGELIS, R.C. Fome oculta: impacto para a população do Brasil. São Paulo : Atheneu, 1999. p.163.

PHILIPPI, S.T., LATTERZA, A.R., CRUZ, A.T.R., RIBEIRO, L.C. Pirâmide alimentar adaptada: guia para escolha 
dos alimentos. Revista de Nutrição, Campinas, v.12, n.1, p.65-80, 1999.

PRIORE, S.E. Composição corporal e hábitos alimentares de adolescentes: uma contribuição à interpretação de indicadores do estado nutricional. São Paulo, 1998. Tese (Doutorado) - Universidade Federal de São Paulo, 1998.

RIBEIRO, R.P.P. Indicadores de hábitos dietéticos e aspectos cognitivos e comportamentais relacionados aos distúrbios de conduta alimentar em adolescentes do sexo feminino. Ribeirão Preto, 1999. Tese (Doutorado) - Faculdade de Medicina de Ribeirão Preto, Universidade de São Paulo, 1999.
WILLIAMS, R.L. Use of the eating attitudes test and eating disorder inventory in adolescents. Journal of Adolescent Health Care, New York, v.8, n.3, p.266272, 1987.

WLODARCZYK-BISAGA, K., DOLAN, B. A two-stage epidemiological study of abnormal eating attitudes and their prospective risk factors in Polish schoolgirls. Psychological Medicine, v.26, n.5, p.1021-1032, 1996.

Recebido para publicação em 10 de maio e aceito em 28 de novembro de 2001. 